\title{
BOUNDING BASIC CHARACTERISTICS OF SPATIAL EPIDEMICS WITH A NEW PERCOLATION MODEL
}

\author{
RONALD MEESTER, ${ }^{*} V U$ University Amsterdam \\ PIETER TRAPMAN, ${ }^{* *}$ VU University Amsterdam and University Medical Center Utrecht
}

\begin{abstract}
We introduce a new 1-dependent percolation model to describe and analyze the spread of an epidemic on a general directed and locally finite graph. We assign a two-dimensional random weight vector to each vertex of the graph in such a way that the weights of different vertices are independent and identically distributed, but the two entries of the vector assigned to a vertex need not be independent. The probability for an edge to be open depends on the weights of its end vertices, but, conditionally on the weights, the states of the edges are independent of each other. In an epidemiological setting, the vertices of a graph represent the individuals in a (social) network and the edges represent the connections in the network. The weights assigned to an individual denote its (random) infectivity and susceptibility, respectively. We show that one can bound the percolation probability and the expected size of the cluster of vertices that can be reached by an open path starting at a given vertex from above by the corresponding quantities for independent bond percolation with a certain density; this generalizes a result of Kuulasmaa (1982). Many models in the literature are special cases of our general model.
\end{abstract}

Keywords: Dependent percolation; epidemics

2010 Mathematics Subject Classification: Primary 60K35; 92D30

\section{Introduction, background, and main results}

We consider an extension of the standard SIR (susceptible $\rightarrow$ infectious $\rightarrow$ removed) epidemic [1], [2], [11] on a directed graph $G=(V, E)$. Here $V$ is the (countable) vertex set of the graph, and $E$ consists of directed edges between vertices in $V$. An edge from $u$ to $v$ is denoted by $u v$, and we say that $v$ is a (directed) neighbor of $u$. We assume that the graph $G=(V, E)$ is simple, that is, for any $u, v \in V$, there is at most one edge from $u$ to $v$. This simplicity assumption can easily be dropped. Furthermore, we assume that the graph is locally finite, in the sense that both the in-degree and out-degree of every vertex are finite.

In a standard SIR epidemic, a vertex is identified with an individual which makes (asymmetric) contacts with each of its neighbors at rate $\tau$. If an infectious individual $u$ contacts a susceptible individual $v$, then $v$ becomes infectious itself. If an individual becomes infectious, it will stay infectious for a random time; the infectious periods of different individuals are independent and identically distributed (i.i.d.). After the infectious period an individual is removed, which can either mean that it is recovered or that the individual has died. A removed

Received 16 March 2009; revision received 8 September 2010.

* Postal address: Department of Mathematics, VU University Amsterdam, De Boelelaan 1081a, 1081 HV Amsterdam, The Netherlands.

** Current address: Department of Mathematics, Stockholm University, 10691 Stockholm, Sweden.

Email address: ptrapman@math.su.se 
individual never becomes susceptible or infectious again. Usually, we assume that there is one initially infectious individual $v_{0}$, and that all other individuals in the network are initially susceptible. Furthermore, we assume that demography plays no role, in the sense that we ignore births, deaths not caused by the infectious disease, and migration. This is a reasonable assumption if we consider emerging infectious diseases for which the timescale of the spread is much smaller than the timescale of demography.

In the model just described, we implicitly assume that all individuals in the network are the same (at least with respect to the epidemic) apart from their position in the network. In particular, all individuals will have the same joint distribution of the total infectivity and susceptibility. In real life, however, infectivity and susceptibility show individual variation, notably because of immunological polymorphism, or due to polymorphic reactions to vaccination [4], [5]. The infectivity and susceptibility of one individual are in general not independent. Dependencies may arise because of confounding factors, such as age, general health status, or in the case of sexually transmitted diseases, promiscuity and levels of condom use, which affect the susceptibility and infectivity in the same direction.

In this paper we model heterogeneity of the population by assigning a random infectivity $W_{v}$ and susceptibility $\bar{W}_{v}$ to each vertex $v$ in $V$, where the vectors $\left(W_{v}, \bar{W}_{v}\right)$ are assumed to be i.i.d. and distributed as $(W, \bar{W})$, taking values in a convex subset $S$ of $\mathbb{R}_{+}^{2}:=[0, \infty)^{2}$. We do not assume that $W_{v}$ and $\bar{W}_{v}$ are independent. Conditionally on the weights, if $u$ becomes infected and $u v \in E$, then $v$ becomes infected (if it was not already) with probability $\kappa\left(W_{u}, \bar{W}_{v}\right)$, where $\kappa$ is some connection function, specified in the model. In percolation terms, this means that the directed edge $u v$ is open with (conditional) probability $\kappa\left(W_{u}, \bar{W}_{v}\right)$. Conditioned on the weights, the states of the edges are independent. However, without this conditioning, states of edges sharing an end vertex are dependent through the weights assigned to this common end vertex.

We are mainly interested in (i) the probability of a large outbreak, (ii) the probability that a disease spreads from one given individual to another, and (iii) the expected final size of an epidemic. Percolation models have served before as useful tools to analyze these quantities; see, for instance, [10], [14], [15], [16], [17], and [19] for related material.

We denote by $\mathrm{P}$ the probability measure governing the full process of assigning weights, and making the edges open or closed. (We do not really need to formally define the full sample space of the process.) One necessary property is that

$$
\mathrm{P}\left(\kappa\left(W_{u}, \bar{W}_{v}\right) \in[0,1]\right)=1,
$$

since $\kappa(\cdot, \cdot)$ represents a probability. Sometimes it is useful to discuss the induced measure of $\mathrm{P}$ on the space $\Omega:=$ \{open, closed ${ }^{E}$, that is, the induced measure on configurations of open and closed edges.

We consider connection functions $\kappa(x, y)$, which can be written as $\kappa(x y)$, where $\kappa(z)$ is nondecreasing and concave. Examples of functions satisfying these conditions are

$$
\begin{gathered}
\kappa_{a}(x, y)=x y \quad \text { with } S=[0,1]^{2}, \\
\kappa_{b}(x, y)=1-\mathrm{e}^{-\alpha x y} \quad \text { with } \alpha>0 \text { and } S=\mathbb{R}_{+}^{2}, \\
\kappa_{c}(x, y)=x y(\beta+x y)^{-1} \quad \text { with } \beta>0 \text { and } S=\mathbb{R}_{+}^{2} .
\end{gathered}
$$

We note that if $\kappa(x, y)$ is factorizable, i.e. if there exist functions $\kappa_{1}(x)$ and $\kappa_{2}(y)$ such that $\kappa(x, y)=\kappa_{1}(x) \kappa_{2}(y)$, then we can without loss of generality assume that $\kappa(x, y)=x y$ and $S=[0,1]^{2}$. This can easily be seen by first replacing $W$ by $\kappa_{1}(W)$ and $\bar{W}$ by $\kappa_{2}(\bar{W})$, and then 
scaling $W$ and $\bar{W}$ such that they both take values in $[0,1]$ with probability 1 . This is possible since, by (1.1) and the independence of $W_{u}$ and $\bar{W}_{v}$ for $u \neq v$, there exists a constant $c>0$ such that $\mathrm{P}\left(\kappa_{1}(W) \leq c\right)=\mathrm{P}\left(\kappa_{2}(\bar{W}) \leq 1 / c\right)=1$.

The connection functions $\kappa_{a}, \kappa_{b}$, and $\kappa_{c}$ have important epidemiological interpretations. A factorizable connection function is appropriate in situations in which there is at most one contact from an infectious individual to a given neighbor during its infectious period, or when only at the first contact of an infectious individual with a given neighbor the infection may be transmitted. This last assumption is proposed in some models for the spread of HIV [20], where the number of sexual contacts per couple can be ignored and only the number of partners is of importance. In those models the probability of an infectious contact from $u$ to $v$ is given by $\kappa\left(W_{u}, \bar{W}_{v}\right)=W_{u} \bar{W}_{v}$, with $W_{u}=1-\exp \left[-\int_{0}^{\Lambda_{u}} \tau_{u}(x) \mathrm{d} x\right]$, where $\Lambda_{u}$ is the length of the infectious period of individual $u$ and $\tau_{u}(x)$ is the (possibly inhomogeneous) rate at which individual $u$ makes infectious contacts at time $x$ after its infection, and where $\bar{W}_{v}$ is the probability that individual $v$ (if still susceptible) becomes infected at an infectious contact.

The choice in (1.2) can be found in [18], and (1.3) is discussed in [7], both in the context of complete graphs. In neither of these two papers are epidemiological interpretations of the connection functions given. To shed some light on a possible epidemiological interpretation of $\kappa_{b}$ and $\kappa_{c}$, we write

$$
\kappa_{b}(x, y)=1-\mathrm{e}^{-\alpha x y}=1-\sum_{k=0}^{\infty} \frac{(\alpha x)^{k}}{k !} \mathrm{e}^{-\alpha x}(1-y)^{k}
$$

and

$$
\kappa_{c}(x, y)=\frac{x y}{\beta+x y}=1-\sum_{k=0}^{\infty} \frac{\beta}{x+\beta}\left(\frac{x}{x+\beta}\right)^{k}(1-y)^{k} .
$$

When $0<y<1$, we see that we can interpret these connection functions as follows. For $\kappa_{c}$, an infectious individual has a geometric- $\beta /(x+\beta)$ number of contacts with a neighbor, and each time, the probability that the infection is accepted is equal to $y$. For $\kappa_{b}$, a similar interpretation is possible, replacing the geometric number of attempts by a Poisson- $\alpha x$ number. In both cases, the number of attempts stochastically increases when $x$ grows, in accordance to our interpretation of $W$ as the infectivity.

It should be noted that (1.3) arises when the infectious period of every individual is exponentially distributed with parameter $\beta$ and the per neighbor infection rate of individual $u$ is $W_{u}$, while the probability that an infectious contact with susceptible individual $v$ leads to an infection is given by $\bar{W}_{v}$. If the infectious period of individual $u$ is $\Lambda_{u}$, and during its infectious period it makes infectious contacts with every neighbor at rate $\alpha \tau_{u}$, then the number of attempts will have a Poisson- $\alpha \tau_{u} \Lambda_{u}$ distribution; hence, both $\kappa_{b}$ and $\kappa_{c}$ arise naturally. Note that replacing the combination $\kappa_{c}$ and $(W, \bar{W})$ by $\kappa_{b}$ and $(\Lambda W / \alpha, \bar{W})$, where $\Lambda$ is exponentially distributed with parameter $\beta$ and independent of $W$, does not change the induced measure on $\Omega$.

We define the usual independent bond percolation measure $\mathrm{P}_{p}^{\text {bond }}$ as the product measure on $\Omega$ in which edges are independently open with probability $p$ [12]. If $\kappa(x, y)=x y$, $\mathrm{P}\left(W=w^{*}, \bar{W}=\bar{w}^{*}\right)=1$, and $w^{*} \bar{w}^{*}=p$, then the induced measure of $\mathrm{P}$ on $\Omega$ is just $\mathrm{P}_{p}^{\text {bond }}$. If $\kappa(x, y)=x y$ and $\mathrm{P}(W=\bar{W}=1)=1-\mathrm{P}(W=\bar{W}=0)=p$, we denote the corresponding measure by $\mathrm{P}_{p}^{\text {site }}$. Indeed, this measure corresponds to the edge representation of an independent site percolation model with parameter $p$ [12], in which an edge is open if and only if both its starting and ending vertices are open. Note that although $\mathrm{P}_{p}^{\text {bond }}$ is defined on $\Omega, \mathrm{P}_{p}^{\text {site }}$ is defined on the full space. This is perhaps slightly confusing, but it works best this way. 
In order to state our results, we need a few definitions. An ordered set of edges in $E$, $\xi=\left(v_{0} v_{1}, v_{1} v_{2}, \ldots, v_{n-1} v_{n}\right)$, is a (directed) path of length $n$ from $v_{0}$ to $v_{n}$. If $v_{i} \neq v_{j}$ for all $0 \leq i<j \leq n$ then the path is self-avoiding. It is straightforward to extend this definition to self-avoiding paths of infinite length. If we assume that a path is self-avoiding, we will explicitly mention this. With some abuse of terminology we define a trivial path as a path without edges, but with a starting vertex and the same end vertex (i.e. a trivial path may be seen as a single vertex).

We say that a path is open if all edges in the path are open; a trivial path is always open. We use the notation $v_{i} \rightsquigarrow v_{j}$ if there is at least one open path from $v_{i}$ to $v_{j}$. If the final vertex of a path $\xi_{1}$ is the first vertex of a path $\xi_{2}$, we write $\left(\xi_{1}, \xi_{2}\right)$ for the conjunction of $\xi_{1}$ and $\xi_{2}$, that is, if $\xi_{1}=\left(v_{0} v_{1}, v_{1} v_{2}, \ldots, v_{n-1} v_{n}\right)$ and $\xi_{2}=\left(v_{n} v_{n+1}, v_{n+1} v_{n+2}, \ldots, v_{n+m-1} v_{n+m}\right)$, then

$$
\left(\xi_{1}, \xi_{2}\right)=\left(v_{0} v_{1}, v_{1} v_{2}, \ldots, v_{n-1} v_{n}, v_{n} v_{n+1}, \ldots, v_{n+m-1} v_{n+m}\right)
$$

For a finite or infinite path $\xi=\left(v_{0} v_{1}, v_{1} v_{2}, \ldots, v_{n-1} v_{n}, v_{n} v_{n+1}, \ldots\right)$, we define the truncation of $\xi$ after $n$ edges as $\xi^{S}(n):=\left(v_{0} v_{1}, v_{1} v_{2}, \ldots, v_{n-1} v_{n}\right)$. The tail of $\xi$ starting after $n$ edges is defined as $\xi^{t}(n):=\left(v_{n} v_{n+1}, \ldots\right)$. Both the truncation after $n$ edges and the tail starting after $n$ edges may be trivial paths. We are now ready to specify the collections of paths we consider in this paper.

Definition 1.1. We say that a collection of paths $\Xi$ is weakly hoppable if, for any $v \in V$, any $i, j \in \mathbb{N}$, and any two paths $\xi, \phi \in \Xi$ going through $v$, where $v$ is the end vertex of the $i$ th edge of $\xi$ and the start vertex of the $j$ th edge of $\phi$, the conjunction $\left(\xi^{s}(i), \phi^{t}(j)\right)$ is in $\Xi$ as well.

In words, we need to be able to 'hop' from one path to another if they cross. We allow for $\xi=\phi$, which implies that if a path in a weakly hoppable collection of paths $\Xi$ has a loop then this loop can be erased and the resulting path is still in $\Xi$.

Furthermore, let $E^{(n)}$ be the collection of the first $n$ edges in $E$, according to some given enumeration of the edges for which $\bigcup_{n \in \mathbb{N}} E^{(n)}=E$. We 'approximate' $\Xi$ by sets $\Xi_{n}$ defined as follows: $\Xi_{n}$ is the collection of all infinite paths which start in $E^{(n)}$ truncated at the first instance they leave $E^{(n)}$ together with all finite paths of which all edges are in $E^{(n)}$. If the first edge of an infinite path $\xi$ is not in $E^{(n)}$ then the trivial path at the starting vertex of $\xi$ is in $\Xi_{n}$.

Finally, for a collection of paths $\Xi$ we denote by $\mathcal{C}^{\Xi}$ the event that at least one path in $\Xi$ is open. We note that, for a weakly hoppable collection of paths $\Xi, \mathcal{C}^{\Xi}$ is equivalent to the event that at least one self-avoiding path in $\Xi$ is open, because loop-erased paths from $\Xi$ are also in $\Xi$.

Definition 1.2. We say that a collection of paths $\Xi$ is hoppable if it is weakly hoppable and if in addition, for some enumeration of the edges,

$$
e^{\Xi}=\lim _{n \rightarrow \infty} e^{\Xi_{n}}
$$

Remark 1.1. 1. Most natural and useful collections of paths are hoppable. For instance, the collection of infinite paths starting at a given vertex (or, more generally, in a finite set) is hoppable, as is the collection of all paths from a given vertex $u$ to a given vertex $v$ (or, more generally, from a finite set to another finite set). To see the first claim, we note that if $\Xi$ is the collection of all infinite paths starting at a finite set then $\mathcal{C}^{\Xi_{n+1}} \subset \mathcal{C}^{\Xi_{n}}$ for all $n$ and $\mathcal{C}^{\Xi}=\bigcap_{n=1}^{\infty} \mathcal{C}^{\Xi_{n}}$. If $\Xi$ is the collection of all paths from a finite set to another finite set then $\mathcal{C}^{\Xi_{n}} \subset \mathcal{C}^{\Xi_{n+1}}$ for all $n$ and $\mathcal{C}^{\Xi}=\bigcup_{n=1}^{\infty} \mathcal{C}^{\Xi_{n}}$. 
2. If $\Xi_{n}$ had been defined as 'the collection of all paths which start in $E^{(n)}$, truncated at the first instance they leave $E^{(n)}$, , then the collection of all paths from vertex $u$ to vertex $v$ would not have been hoppable. Indeed, on an infinite graph we have $\mathcal{C}^{\Xi_{n+1}} \subset \mathcal{C}^{\Xi_{n}}$ for all $n$, but $\mathcal{C}^{\Xi} \neq \bigcap_{n=1}^{\infty} \mathcal{C}^{\Xi_{n}}$, since $\bigcap_{n=1}^{\infty} \mathcal{C}^{\Xi_{n}}$ occurs if there is an open path from $u$ to $v$ or if there is an infinite open path starting at $u$.

The main results of this paper are the following, generalizing results in [15] (see also [16] for related results for the case in which $W$ and $\bar{W}$ are independent).

Theorem 1.1. Let $(W, \bar{W})$ be a random vector taking values in $S$, and let $\kappa(x, y)=\kappa(x y)$ be such that $\kappa(z)$ is increasing and concave. Then, for

$$
p=\kappa(\max [\mathrm{E}(W \bar{W}), \mathrm{E}(W) \mathrm{E}(\bar{W})])
$$

and any hoppable collection of paths $\Xi$, we have

$$
\mathrm{P}\left(\mathcal{C}^{\Xi}\right) \leq \mathrm{P}_{p}^{\text {bond }}\left(\mathcal{C}^{\Xi}\right)
$$

Theorem 1.2. Let $\kappa(x, y)=x y$ and $S=[0,1]^{2}$. Then, for $p=\mathrm{E}(W \bar{W})$ and any hoppable collection $\Xi$ of paths in $E$, we have

$$
\mathrm{P}\left(\mathcal{C}^{\Xi}\right) \geq \mathrm{P}_{p}^{\mathrm{site}}\left(\mathcal{C}^{\Xi}\right)
$$

Theorems 1.1 and 1.2 are corollaries of Theorem 3.1 below.

We note that if $E$ is symmetric, i.e. $u v \in E$ is equivalent to $v u \in E$, and if $\mathrm{P}(W=\bar{W})=1$ and $\kappa(x, y)=\kappa(y, x)$, then the law of the cluster of vertices that can be reached by open paths from $v \in V$ on $G$ is the same as the law of the open cluster containing $v$ on the undirected counterpart of $G$ (the graph obtained by replacing the two edges connecting the same vertices by one undirected edge) [10]. Hence, many questions on undirected graphs can be addressed as questions on directed graphs, and Theorems 1.1 and 1.2 may be applied to percolation on undirected graphs as well.

We will illustrate the results by a numerical example, and then by discussing the situation on a tree. In this latter example, we also shed some light on the reason why $\mathrm{E}(W \bar{W})$ and $\mathrm{E}(W) \mathrm{E}(\bar{W})$ play an important role in our analysis.

Example 1.1. Suppose that $W=\bar{W}$ is uniformly distributed on $(a, 1)$ for some $a \in(0,1)$, $G=\mathbb{L}^{2}$ is the square lattice with nearest neighbor (directed) edges and $\kappa(x, y)=x y$. If $a \leq \sqrt{\frac{3}{4}}-\frac{1}{2} \approx 0.37$ then $\mathrm{E}(W \bar{W})=\mathrm{E}\left(W^{2}\right) \leq \frac{1}{2}$. Because the critical value for independent bond percolation is $\frac{1}{2}$ [12], in view of the remark above on the relation between percolation on directed and undirected graphs, the probability that the cluster of individuals that can be reached by an open path from the origin is infinite is 0 for this model. Let $p_{\mathrm{c}}^{\text {site }}\left(\mathbb{L}^{2}\right) \approx 0.59$ be the critical value for site percolation on $\mathbb{L}^{2}$. If $a>\frac{1}{2}\left(\sqrt{12 p_{\mathrm{c}}^{\text {site }}\left(\mathbb{L}^{2}\right)-3}-1\right) \approx 0.51$ then the probability that the cluster of individuals that can be reached by an open path from the origin is infinite is positive for this model.

Example 1.2. Let $G=(V, E)$ be the rooted tree in which all vertices have out-degree $d$ and in-degree 1 , apart from the root $v_{0}$ which has in-degree 0 . We say that the root is the generation- 0 vertex; if there is a path of length $n$ from the root to vertex $v$, then $v$ is a said to be a generation- $n$ vertex.

Let $\kappa(x, y)=x y$, and let $\Xi_{k}$ be the set of all paths of length $k$ starting at the root. Furthermore, let $Z_{k}$ be the number of open paths in $\Xi_{k}$, i.e. $Z_{k}$ is the number of generation- $k$ 
vertices that can be reached by an open path from the root. For $k \geq 1$ and generation- 1 vertex $v_{1}$, we define $Y_{k}\left(v_{1}\right)$ as the number of generation- $k$ vertices $v_{k}$, for which there is an open path from $v_{0}$ to $v_{k}$ through $v_{1}$, conditioned on $W_{v_{0}}=1$ and $\bar{W}_{u}=1$ for all generation- $k$ vertices $u$.

We claim that $Y_{k}\left(v_{1}\right)$ is distributed as the size of the $(k-1)$ th generation of a Galton-Watson process [13] starting with one individual, in which individuals have no offspring with probability $\mathrm{E}(1-\bar{W})+\mathrm{E}\left(\bar{W}(1-W)^{d}\right)$ and offspring of size $j$ with probability $\mathrm{E}\left(\bar{W}\left(\begin{array}{l}d \\ j\end{array}\right) W^{j}(1-W)^{d-j}\right)$ for $0<j \leq d$. Indeed, we can adopt the point of view that, for a vertex to have any offspring, it first has to accept the disease from its predecessor in the tree, and, if it does, in addition it needs to send the disease to the next generation. This leads to an offspring mean of $d \mathrm{E}(\bar{W} W)$.

It now seems quite natural, given the computation above, to consider a class of probability measures for which $\mathrm{E}(W \bar{W})$ is constant (compare [4] and [5]). However, in order to prove the inequality $\mathrm{P}\left(\mathcal{C}^{\Xi}\right) \leq \mathrm{P}_{p}^{\text {bond }}\left(\mathcal{C}^{\Xi}\right)$ for all hoppable collections of paths in $E$, we need the additional assumption that $p \geq \mathrm{E}(W) \mathrm{E}(\bar{W})$. This can be seen by assuming that $\Xi$ consists of a single edge. The marginal probability that this edge is open is given by $\mathrm{E}(W) \mathrm{E}(\bar{W})$. This explains, to some extent, the importance of the quantities $\mathrm{E}(W \bar{W})$ and $\mathrm{E}(W) \mathrm{E}(\bar{W})$.

\section{Discussion}

Before we start proving the results, we collect in this section a number of remarks.

- Contrary to Kuulasmaa [15] and Miller [16], we deal with infectious diseases for which the susceptibility and infectivity of individuals might be dependent. This dependence complicates the proofs and means that we cannot apply the results from [15] and [16] immediately. If $\kappa(x, y)$ is not factorizable then Miller's result for independent $W$ and $\bar{W}$ is slightly stronger than our result. This is because he provided a bond percolation upper bound with parameter $p=\mathrm{E}(\kappa(W \bar{W}))$, which, for concave $\kappa$, is bounded above by our bond percolation parameter $\kappa(\mathrm{E}(W \bar{W}))$.

- The class of possible collections $\Xi$ for which the result is true is larger than the class of hoppable collections. We have chosen this formulation because it contains most collections of interest, and also because of its elegance. One class of paths that can be seen to satisfy the results is the class of infinite backwards paths ending at a given vertex $v$. Indeed, by simply interchanging the role of $W$ and $\bar{W}$ (see also [16] for this trick), it is easy to see that our results are valid for this class as well.

- Suppose that $\kappa(x, y)=x y$. By choosing $\Xi$ as the collection of paths from $u$ to $v$, we find that among all measures with $\mathrm{E}(W \bar{W}) \geq \mathrm{E}(W) \mathrm{E}(\bar{W})$, it is the case that $\mathrm{E}[1(u \rightsquigarrow v)]=$ $\mathrm{P}(u \rightsquigarrow v)$ is at most $\mathrm{P}_{\mathrm{E}(W \bar{W})}^{\text {bond }}(u \rightsquigarrow v)$ and at least $\mathrm{P}_{\mathrm{E}(W \bar{W})}(u \rightsquigarrow v)$. Let $C_{u}$ be the set of vertices that can be reached by an open path from vertex $u$. The observations above give

$$
\mathrm{E}\left(\left|C_{u}\right|\right)=\sum_{v \in V} \mathrm{P}(u \rightsquigarrow v) \leq \sum_{v \in V} \mathrm{P}_{\mathrm{E}(W \bar{W})}^{\text {bond }}(u \rightsquigarrow v)=\mathrm{E}_{\mathrm{E}(W \bar{W})}^{\text {bond }}\left(\left|C_{u}\right|\right) .
$$

- It is not possible to use straightforward stochastic domination arguments to prove the theorems in their full generality. This can be seen by considering $\kappa(x, y)=x y$, and uncorrelated $W$ and $\bar{W}$. In that case the marginal probability that any edge $u v \in E$ is open is the same for all measures for which $\mathrm{E}(W \bar{W})$ is constant. However, the edge density in $\mathrm{P} \mathrm{E}(W \bar{W})$ is also $\mathrm{E}(W \bar{W})$ and, hence, stochastic domination cannot be used to prove Theorem 1.1 . 
- We can compare some of our results with bounds given in [3]: any undirected 1-dependent edge percolation model on the two-dimensional square lattice, with marginal probability for an edge to be open at least 0.8639 , is supercritical. Since this bound holds for all 1-dependent measures, it is to be expected that our bounds improve on this in our specific model. Since we can only compare undirected models, we can only compare in the symmetric case (which is perhaps not the most interesting case). Still, since $\mathrm{P}_{p}^{\text {site }}$ percolates above $p=0.68$ [21] (this is a rigorous bound, the correct value of the critical probability is around 0.59), we see that our model percolates when $\mathrm{P}(W=\bar{W})=1$, $\kappa(x, y)=x y$, and $\mathrm{E}(W \bar{W})=\mathrm{E}\left(W^{2}\right) \geq 0.68$. Hence, we improve on the general bound when $\mathrm{E}\left(W^{2}\right) \geq 0.68$ and $(\mathrm{E}(W))^{2}<0.8639$.

- Our model is a generalization of many other percolation processes, such as the locally dependent random graph model [15], mixed percolation [8], generalized random graphs [7], and Poissonian random graphs [18], where the two latter ones were previously defined for only finite complete graphs $G$. The inhomogeneous random graphs of [6] are defined only on complete graphs, but they are more general than our model on the complete graph.

\section{Proofs}

Let $S_{1}$ be the projection of $S$ in the first coordinate direction, and let $S_{2}$ be the projection of $S$ in the second coordinate direction. Let $E_{v}^{\prime}$ be the set of all edges starting at $v$, and let $E_{v}^{*}$ be the set of all edges ending at $v$. For any pair of (possibly empty) sets $A \subset E_{u}^{\prime}$ and $B \subset E_{u}^{*}$, any $|A|$-dimensional vector $\boldsymbol{x}=\left(x_{1}, x_{2}, \ldots, x_{|A|}\right) \in\left(S_{2}\right)^{|A|}$, and any $|B|$-dimensional vector $\boldsymbol{y}=$ $\left(y_{1}, y_{2}, \ldots, y_{|B|}\right) \in\left(S_{1}\right)^{|B|}$, we define the zero function $z_{u}(\mathrm{P} ; A, B ; \boldsymbol{x}, \boldsymbol{y})$ as the probability that either none of the edges in $A$ are open or none of the edges in $B$ are open if the weights assigned to the endpoints of the edges in $A$ and $B$ are the elements of the vectors $\boldsymbol{x}$ and $\boldsymbol{y}$, respectively. This can be expressed as

$$
z_{u}(\mathrm{P} ; A, B ; \boldsymbol{x}, \boldsymbol{y}):=\mathrm{E}\left(1-\left[1-\prod_{i=1}^{|A|}\left(1-\kappa\left(W, x_{i}\right)\right)\right]\left[1-\prod_{j=1}^{|B|}\left(1-\kappa\left(y_{j}, \bar{W}\right)\right)\right]\right)
$$

if $|A||B|>0$. For the remaining cases, we define

$$
\begin{aligned}
& z_{u}(\mathrm{P} ; A, \varnothing ; \boldsymbol{x}, \varnothing):=\mathrm{E}\left(\prod_{i=1}^{|A|}\left(1-\kappa\left(W, x_{i}\right)\right)\right) \quad \text { if }|A|>0, \\
& z_{u}(\mathrm{P} ; \varnothing, B ; \varnothing, \boldsymbol{y}):=\mathrm{E}\left(\prod_{j=1}^{|B|}\left(1-\kappa\left(y_{j}, \bar{W}\right)\right)\right) \quad \text { if }|B|>0, \\
& z_{u}(\mathrm{P} ; \varnothing, \varnothing ; \varnothing, \varnothing):=1 .
\end{aligned}
$$

If the graph is transitive, we do not need the reference to the vertex $u$ in the zero function.

In the epidemiological setting, the zero function $z_{u}(\mathrm{P} ; A, B ; \boldsymbol{x}, \boldsymbol{y})$ is the (conditional) probability that if all endpoints of edges in $B$ become infected and have ' $W$-weights' $y_{1}, \ldots, y_{|B|}$, either $u$ will not get infected via an edge in $B$, or $u$ will not transmit the disease to any of the endpoints of edges in $A$, if those endpoints have ' $\bar{W}$-weights' $x_{1}, \ldots, x_{|A|}$. 
We write $z_{v}\left(\mathrm{P}^{(a)}\right) \geq z_{v}\left(\mathrm{P}^{(b)}\right)$ if $z_{v}\left(\mathrm{P}^{(a)} ; A, B ; \boldsymbol{x}, \boldsymbol{y}\right) \geq z_{v}\left(\mathrm{P}^{(b)} ; A, B ; \boldsymbol{x}, \boldsymbol{y}\right)$ for all $A \subset E_{v}^{\prime}$, all $B \subset E_{v}^{*}$, all $\boldsymbol{x} \in\left(S_{2}\right)^{|A|}$, and all $\boldsymbol{y} \in\left(S_{1}\right)^{|B|}$. The following result is interesting in its own right, and will be the main tool to prove Theorem 1.1.

Theorem 3.1. If the distributions of the weights with respect to the measures $\mathrm{P}^{(a)}$ and $\mathrm{P}^{(b)}$ are such that $z_{v}\left(\mathrm{P}^{(a)}\right) \leq z_{v}\left(\mathrm{P}^{(b)}\right)$ for all $v \in V$ then, for any hoppable collection $\Xi$ of paths,

$$
\mathrm{P}^{(b)}\left(\mathcal{C}^{\Xi}\right) \leq \mathrm{P}^{(a)}\left(\mathcal{C}^{\Xi}\right)
$$

Remark 3.1. Theorem 3.1 does not hold if we would allow for all collections of paths $\Xi$. Here is a counterexample. Let $G$ be the subgraph of the two-dimensional square lattice, consisting of the origin and its nearest neighbors (with nearest neighbor edges). Let $\kappa(x, y)=x y$, and suppose that the weights assigned to the neighbors of the origin are all equal to 1 . We consider two measures $\mathrm{P}^{(a)}$ and $\mathrm{P}^{(b)}$ on the weights assigned to the origin: $\mathrm{P}^{(a)}(W=\bar{W})=1$, and $W$ is uniformly $(0,1)$ distributed under $\mathrm{P}^{(a)}$, while $\mathrm{P}^{(b)}(W=\bar{W}=0)=\frac{17}{48}, \mathrm{P}^{(b)}\left(W=\bar{W}=\frac{2}{3}\right)=$ $\frac{9}{16}$, and $\mathrm{P}^{(b)}(W=\bar{W}=1)=\frac{1}{12}$.

Now let $\xi$ be the path from $(0,-1)$ to $(0,1)$, let $\phi$ be the path from $(-1,0)$ to $(1,0)$ in $G$, and let $\Xi=\{\xi, \phi\}$; this is not a hoppable collection. A quick computation gives

$$
\mathrm{P}^{(a)}\left(\mathcal{C}^{\Xi}\right)=\frac{7}{15}<\frac{17}{36}=\mathrm{P}^{(b)}\left(\mathcal{C}^{\Xi}\right)
$$

All weights of neighbors of the origin are 1 , so we only have to consider $z_{0}\left(\mathrm{P}^{(a)} ; A, B\right):=$ $z_{0}\left(\mathrm{P}^{(a)} ; A, B ; \mathbf{1}, \mathbf{1}\right)$ and $z_{0}\left(\mathrm{P}^{(b)} ; A, B\right):=z_{0}\left(\mathrm{P}^{(b)} ; A, B ; \mathbf{1}, \mathbf{1}\right)$, where $\mathbf{1}$ is the vector with all entries equal to 1 . It follows from some tedious but straightforward computations that $z_{0}\left(\mathrm{P}^{(a)}\right) \leq z_{0}\left(\mathrm{P}^{(b)}\right)$; we do not present the computation here.

In the proof of Theorem 3.1 we need the following lemma.

Lemma 3.1. If $\Xi$ is hoppable then $\Xi_{n}$ is hoppable for all $n \in \mathbb{N}$.

Proof. Since $\Xi_{n}$ consists of finitely many edges only, it is enough to prove that $\Xi_{n}$ is weakly hoppable, that is, for any $v$ and any two paths $\xi_{n}, \phi_{n} \in \Xi_{n}$ going through $v$ (say that $v$ is the end vertex of the $i$ th edge of $\xi$ and the start vertex of the $j$ th edge of $\phi$ ), the conjunction $\left(\xi_{n}^{s}(i), \phi_{n}^{t}(j)\right)$ is in $\Xi_{n}$ as well. We distinguish various cases.

1. If the $\phi_{n}$ are the truncations of the infinite path $\phi \in \Xi$ then $\left(\xi_{n}^{s}(i), \phi_{n}^{t}(j)\right)$ is the truncation of $\left(\xi_{n}^{S}(i), \phi^{t}(j)\right)$ at the first time this paths leaves $E_{n}$. All of its edges are in $E_{n}$. By the weak hoppability of $\Xi$, this conjunction is in $\Xi_{n}$ as well.

2. If the $\xi_{n}$ are the truncations of the infinite path $\xi \in \Xi$ and $\phi_{n} \in \Xi$, then $\left(\xi_{n}^{s}(i), \phi_{n}^{t}(j)\right)$ contains only edges in $E_{n}$ and is in $\Xi$ by the weak hoppability of $\Xi$ and in $\Xi_{n}$ by the definition of $\Xi_{n}$.

3. If $\xi_{n}$ and $\phi_{n}$ are both finite paths in $\Xi$, then $\left(\xi_{n}^{s}(i), \phi_{n}^{t}(j)\right)$ contains only edges in $E_{n}$ and is in $\Xi$ by the weak hoppability of $\Xi$, and in $\Xi_{n}$ by definition.

Proof of Theorem 3.1. (i) First we assume that there is a $u \in V$ such that $z_{u}\left(\mathrm{P}^{(a)}\right) \leq z_{u}\left(\mathrm{P}^{(b)}\right)$, and that the distribution of $\left(W_{v}, \bar{W}_{v}\right)$ is the same under $\mathrm{P}^{(a)}$ and $\mathrm{P}^{(b)}$ (and, therefore, $z_{v}\left(\mathrm{P}^{(a)}\right)=$ $\left.z_{v}\left(\mathrm{P}^{(b)}\right)\right)$ for all $v \in V \backslash\{u\}$. Under these assumptions, we will now prove that, for all $n \geq 1$, $\mathrm{P}^{(a)}\left(\mathcal{C}^{\Xi_{n}}\right) \geq \mathrm{P}^{(b)}\left(\mathcal{C}^{\Xi_{n}}\right)$.

Let $E_{u}^{(n)}$ be the set of edges in $E^{(n)}$ with $u$ as the start or end vertex. Let $\sigma_{u}^{(n)}$ denote a typical realization of the weights outside $u$, together with the states (open or closed) of the edges in 
$E^{(n)} \backslash E_{u}^{(n)}$. Informally, $\sigma_{u}^{(n)}$ contains all information which does not depend on $\left(W_{u}, \bar{W}_{u}\right)$, and we will proceed by conditioning on $\sigma_{u}^{(n)}$. By assumption, the distribution $\left(W_{v}, \bar{W}_{v}\right)$ is the same with respect to $\mathrm{P}^{(a)}$ and $\mathrm{P}^{(b)}$ for all $v \in V \backslash\{u\}$. This implies that

$$
\mathrm{P}^{(a)}\left(\mathcal{C}^{\Xi_{n}}\right)-\mathrm{P}^{(b)}\left(\mathcal{C}^{\Xi_{n}}\right)=\int\left(\mathrm{P}^{(a)}\left(\mathcal{C}^{\Xi_{n}} \mid \sigma_{u}^{(n)}\right)-\mathrm{P}^{(b)}\left(\mathcal{C}^{\Xi_{n}} \mid \sigma_{u}^{(n)}\right)\right) \mathrm{dP}^{(a)}\left(\sigma_{u}^{(n)}\right) .
$$

We now split the $\sigma_{u}^{(n)}$ into three cases.

1. $\sigma_{u}^{(n)}$ is such that $\mathrm{P}^{(a)}\left(\mathcal{C}^{\Xi_{n}} \mid \sigma_{u}^{(n)}\right)=\mathrm{P}^{(b)}\left(\mathcal{C}^{\Xi_{n}} \mid \sigma_{u}^{(n)}\right)=0$, in which case

$$
\mathrm{P}^{(a)}\left(\mathcal{C}^{\Xi_{n}} \mid \sigma_{u}^{(n)}\right)-\mathrm{P}^{(b)}\left(\mathcal{C}^{\Xi_{n}} \mid \sigma_{u}^{(n)}\right)=0 .
$$

This case occurs if $\sigma_{u}^{(n)}$ is such that no matter the weights assigned to $u$, the probability of an open path in $\Xi_{n}$ is 0 .

2. $\sigma_{u}^{(n)}$ is such that $\mathrm{P}^{(a)}\left(\mathcal{C}^{\Xi_{n}} \mid \sigma_{u}^{(n)}\right)=\mathrm{P}^{(b)}\left(\mathcal{C}^{\Xi_{n}} \mid \sigma_{u}^{(n)}\right)=1$, in which case

$$
\mathrm{P}^{(a)}\left(\mathcal{C}^{\Xi_{n}} \mid \sigma_{u}^{(n)}\right)-\mathrm{P}^{(b)}\left(\mathcal{C}^{\Xi_{n}} \mid \sigma_{u}^{(n)}\right)=0 .
$$

This case occurs if $\sigma_{u}^{(n)}$ is such that no matter the weights assigned to $u$, the probability of an open path in $\Xi_{n}$ is 1 .

3. The only case remaining is that $\sigma_{u}^{(n)}$ is such that neither

$$
\mathrm{P}^{(a)}\left(\mathcal{C}^{\Xi_{n}} \mid \sigma_{u}^{(n)}\right)=\mathrm{P}^{(b)}\left(\mathcal{C}^{\Xi_{n}} \mid \sigma_{u}^{(n)}\right)=1
$$

nor

$$
\mathrm{P}^{(a)}\left(\mathcal{C}^{\Xi_{n}} \mid \sigma_{u}^{(n)}\right)=\mathrm{P}^{(b)}\left(\mathcal{C}^{\Xi_{n}} \mid \sigma_{u}^{(n)}\right)=0 .
$$

In this last case there are three possibilities.

(i) If $u$ is the starting vertex of some path in $\Xi_{n}$ then-since $\Xi_{n}$ is weakly hoppable-the tail starting at $u$ of a self-avoiding path through $u$ is also in $\Xi_{n}$. Therefore, with the assumptions on $\sigma_{u}^{(n)}, \Xi_{n}$ contains an open path if and only if it contains a self-avoiding open path starting at $u$. Let $A \subset E_{u}^{(n)}$ be the set of starting edges of open paths in $\Xi_{n}$ starting at $u$, if all edges in $E_{u}^{(n)}$ would have been open. We note that, because we need to only consider self-avoiding paths in $\Xi_{n}$, at least one of the paths in $\Xi_{n}$ is open if and only if at least one edge in $A$ is open. This probability is given by 1 minus the zero function. Since, $z_{u}\left(\mathrm{P}^{(a)} ; A, \varnothing ; \boldsymbol{x}, \varnothing\right) \leq z_{u}\left(\mathrm{P}^{(b)} ; A, \varnothing ; \boldsymbol{x}, \varnothing\right)$ for all $A$ and $\boldsymbol{x}$, it follows that

$$
\mathrm{P}^{(a)}\left(\mathcal{C}^{\Xi_{n}} \mid \sigma_{u}^{(n)}\right)-\mathrm{P}^{(b)}\left(\mathcal{C}^{\Xi_{n}} \mid \sigma_{u}^{(n)}\right) \geq 0
$$

(ii) If $u$ is the end vertex of some path in $\Xi_{n}$ then-since $\Xi_{n}$ is weakly hoppable-the truncation at vertex $u$ of a self-avoiding path through $u$ is also in $\Xi_{n}$. Therefore, with the assumptions on $\sigma_{u}^{(n)}, \Xi_{n}$ contains a self-avoiding open path if and only if it contains an open path ending at $u$. Let $B \subset E_{u}^{(n)}$ be the set of end edges of open paths in $\Xi_{n}$ ending at $u$, if all edges in $E_{u}^{(n)}$ would have been open. Since $z_{u}\left(\mathrm{P}^{(a)} ; \varnothing, B ; \varnothing, \boldsymbol{y}\right) \leq$ $z_{u}\left(\mathrm{P}^{(b)} ; \varnothing, B ; \varnothing, \boldsymbol{y}\right)$ for all $B$ and $\boldsymbol{y}$, it follows in the same way as for the previous case that

$$
\mathrm{P}^{(a)}\left(\mathcal{C}^{\Xi_{n}} \mid \sigma_{u}^{(n)}\right)-\mathrm{P}^{(b)}\left(\mathcal{C}^{\Xi_{n}} \mid \sigma_{u}^{(n)}\right) \geq 0
$$


(iii) If $u$ is neither a starting vertex nor an end vertex of any path in $\Xi_{n}$, then let $A$ be the set of starting edges of tails of self-avoiding paths in $\Xi_{n}$, cut off at $u$, for which all edges in the tail are open, if all edges in $E_{u}^{(n)}$ would have been open. Similarly, let $B$ be the set of end edges of truncations of self-avoiding paths in $\Xi_{n}$, cut off at $u$, for which all edges in the truncation are open, if all edges in $E_{u}^{(n)}$ would have been open. Since $\Xi_{n}$ is weakly hoppable, it follows that there is no open path in $\Xi_{n}$ if either all edges in $A$ are closed or all edges in $B$ are closed. By $z_{u}\left(\mathrm{P}^{(a)} ; A, B ; \boldsymbol{x}, \boldsymbol{y}\right) \leq z_{u}\left(\mathrm{P}^{(b)} ; A, B ; \boldsymbol{x}, \boldsymbol{y}\right)$ for all $A, B$, $\boldsymbol{x}$, and $\boldsymbol{y}$, it follows that

$$
\mathrm{P}^{(a)}\left(\mathcal{C}^{\Xi_{n}} \mid \sigma_{u}^{(n)}\right)-\mathrm{P}^{(b)}\left(\mathcal{C}^{\Xi_{n}} \mid \sigma_{u}^{(n)}\right) \geq 0
$$

This concludes the first step of the proof.

(ii) In this second step, we relax the condition that the zero functions differ in one place only. Since the event $\mathcal{C}^{\Xi_{n}}$ depends on the weights of at most $2 n$ vertices in $V$, it is straightforward to construct a sequence of probability measures, $\left(\mathrm{P}^{(i)} ; 1 \leq i \leq 2 n\right)$, such that $\mathrm{P}^{(1)}\left(\mathcal{C}^{\Xi_{n}}\right)=$ $\mathrm{P}^{(a)}\left(\mathcal{C}^{\Xi_{n}}\right)$ and $\mathrm{P}^{(2 n)}\left(\mathcal{C}^{\Xi_{n}}\right)=\mathrm{P}^{(b)}\left(\mathcal{C}^{\Xi_{n}}\right)$, and such that, for $1 \leq i \leq 2 n-1$, the distribution of $\left(W_{v}, \bar{W}_{v}\right)$ with respect to $\mathrm{P}^{(i)}$ and $\mathrm{P}^{(i+1)}$ differ in at most one vertex, say $v_{i}$, for which $z_{v_{i}}\left(\mathrm{P}^{(i)}\right) \leq z_{v_{i}}\left(\mathrm{P}^{(i+1)}\right)$. Repeatedly applying part (i) completes this part of the proof.

(iii) To complete the proof, we simply note that from the definition of hoppable we have, for $i=a, b$,

$$
\mathrm{P}^{(i)}\left(e^{\Xi}\right)=\lim _{n \rightarrow \infty} \mathrm{P}^{(i)}\left(\complement^{\Xi_{n}}\right)
$$

and the result follows.

For the proof of Theorem 1.1, we need the following elementary fact.

Lemma 3.2. Let $f_{i}(x), i=1, \ldots, n$, be convex nonincreasing, nonnegative functions on some domain $D \subset \mathbb{R}$. Then $\prod_{i=1}^{n} f_{i}(x)$ is also a convex nonincreasing, nonnegative function on $D$.

Proof. For $n=2$, observe that, for $0<c<1$ and $y>x$,

$$
\begin{aligned}
c f_{1}(x) f_{2}(x)+(1-c) f_{1}(y) f_{2}(y)= & {\left[c f_{1}(x)+(1-c) f_{1}(y)\right]\left[c f_{2}(x)+(1-c) f_{2}(y)\right] } \\
& +c(1-c)\left[f_{1}(y)-f_{1}(x)\right]\left[f_{2}(y)-f_{2}(x)\right] \\
\geq & f_{1}(c x+(1-c) y) f_{2}(c x+(1-c) y),
\end{aligned}
$$

where in the inequality we have used the fact that both $f_{1}$ and $f_{2}$ are nonincreasing and convex. The proof of the lemma can be completed with induction on $n$-we leave the details to the reader.

Proof of Theorem 1.1. Let $\left(w^{*}, \bar{w}^{*}\right) \in S$ be such that

- $\mathrm{E}(W)=w^{*}, \mathrm{E}(\bar{W})=\bar{w}^{*}$, and $\mathrm{E}(W \bar{W}) \leq w^{*} \bar{w}^{*}$, or

- $\mathrm{E}(W) \leq w^{*}, \mathrm{E}(\bar{W}) \leq \bar{w}^{*}$, and $\mathrm{E}(W \bar{W})=w^{*} \bar{w}^{*}$.

Let $\hat{\mathrm{P}}^{\text {bond }}$ be a measure that satisfies $\hat{\mathrm{P}}^{\text {bond }}\left(W=w^{*}, \bar{W}=\bar{w}^{*}\right)=1$.

We proceed by proving that, for all $A, B, \boldsymbol{x}$, and $\boldsymbol{y}$,

$$
z_{v}(\mathrm{P} ; A, B ; \boldsymbol{x}, \boldsymbol{y}) \geq z_{v}\left(\hat{\mathrm{P}}^{\text {bond }} ; A, B ; \boldsymbol{x}, \boldsymbol{y}\right) .
$$

From Theorem 3.1 and the observation that the induced measure of $\hat{\mathrm{P}}^{\text {bond }}$ on $\Omega$ is just $\mathrm{P}_{p}^{\text {bond }}$, where $p=\kappa\left(w^{*} \bar{w}^{*}\right)$, Theorem 1.1 then follows. 
If $|B|>0$ then $z_{v}(\mathrm{P} ; \varnothing, B ; \varnothing, \boldsymbol{y})=\mathrm{E}\left(\prod_{i=1}^{|B|}\left[1-\kappa\left(y_{i} \bar{W}\right)\right]\right)$. The functions $1-\kappa\left(y_{i} \bar{W}\right)$ are by assumption nonnegative, convex, and decreasing. Therefore, by Lemma 3.2 and Jensen's inequality,

$$
\mathrm{E}\left(\prod_{i=1}^{|B|}\left[1-\kappa\left(y_{i} \bar{W}\right)\right]\right) \geq \prod_{i=1}^{|B|}\left[1-\kappa\left(y_{i} \mathrm{E}(\bar{W})\right)\right] \geq \prod_{i=1}^{|B|}\left[1-\kappa\left(y_{i} \bar{w}^{*}\right)\right],
$$

which is equal to $z_{v}\left(\hat{\mathrm{P}}^{\text {bond }} ; \varnothing, B ; \varnothing, \boldsymbol{y}\right)$. For $|A|>0$, we can use similar arguments to show that

$$
z_{v}(\mathrm{P} ; A, \varnothing ; \boldsymbol{x}, \varnothing) \geq z_{v}\left(\hat{\mathrm{P}}^{\text {bond }} ; A, \varnothing ; \boldsymbol{x}, \varnothing\right) \text {. }
$$

If $|A||B| \geq 1$ then we need to show that

$$
\begin{aligned}
& \mathrm{E}\left(\left(1-\prod_{i=1}^{|A|}\left[1-\kappa\left(W x_{i}\right)\right]\right)\left(1-\prod_{j=1}^{|B|}\left[1-\kappa\left(\bar{W} y_{j}\right)\right]\right)\right) \\
& \quad \leq\left(1-\prod_{i=1}^{|A|}\left[1-\kappa\left(w^{*} x_{i}\right)\right]\right)\left(1-\prod_{j=1}^{|B|}\left[1-\kappa\left(\bar{w}^{*} y_{j}\right)\right]\right) .
\end{aligned}
$$

By the assumption that $\kappa(z)$ is increasing concave and taking values in $[0,1]$, and by Lemma 3.2, it follows that $1-\prod_{i=1}^{|A|}\left[1-\kappa\left(x_{i} u\right)\right]$ is concave and increasing in $u$. By this concavity and $\kappa(0) \geq 0$, it is possible to choose $a:=a(\boldsymbol{x}) \geq 0$ and $b:=b(\boldsymbol{x}) \geq 0$ such that

$$
a+b u \geq 1-\prod_{i=1}^{|A|}\left[1-\kappa\left(x_{i} u\right)\right]
$$

for all $u \in S_{1}$ and $a+b w^{*}=1-\prod_{i=1}^{|A|}\left[1-\kappa\left(x_{i} w^{*}\right)\right]$. Similarly, $1-\prod_{j=1}^{|B|}\left[1-\kappa\left(y_{j} u\right)\right]$ is concave and increasing in $u$, and it is possible to choose $\bar{a}:=\bar{a}(\boldsymbol{y}) \geq 0$ and $\bar{b}:=\bar{b}(\boldsymbol{y}) \geq 0$ such that

$$
\bar{a}+\bar{b} u \geq 1-\prod_{j=1}^{|B|}\left[1-\kappa\left(y_{j} u\right)\right]
$$

for all $u \in S_{1}$ and $\bar{a}+\bar{b} \bar{w}^{*}=1-\prod_{j=1}^{|B|}\left[1-\kappa\left(y_{j} \bar{w}^{*}\right)\right]$.

It follows that

$$
\begin{aligned}
\mathrm{E}(( & \left.\left.1-\prod_{i=1}^{|A|}\left[1-\kappa\left(W x_{i}\right)\right]\right)\left(1-\prod_{j=1}^{|B|}\left[1-\kappa\left(\bar{W} y_{j}\right)\right]\right)\right) \\
& \leq \mathrm{E}((a+b W)(\bar{a}+\bar{b} \bar{W})) \\
& =a \bar{a}+a \bar{b} \mathrm{E}(\bar{W})+\bar{a} b \mathrm{E}(W)+b \bar{b} \mathrm{E}(W \bar{W}) \\
& \leq a \bar{a}+a \bar{b} \bar{w}^{*}+\bar{a} b w^{*}+b \bar{b} w^{*} \bar{w}^{*} \\
& =\left(a+b w^{*}\right)\left(\bar{a}+\bar{b} \bar{w}^{*}\right) \\
& =\left(1-\prod_{i=1}^{\infty}\left[1-\kappa\left(w^{*} x_{i}\right)\right]\right)\left(1-\prod_{j=1}^{|B|}\left[1-\kappa\left(\bar{w}^{*} y_{j}\right)\right]\right)
\end{aligned}
$$

where the second inequality follows from the definitions of $w^{*}$ and $\bar{w}^{*}$. This completes the proof of Theorem 1.1. 
Proof of Theorem 1.2. In order to use Theorem 3.1, we have to show that, for all P, $A, B \subset$ $E_{v}^{(n)}$, all $\boldsymbol{x} \in\left(S_{1}\right)^{|A|}$, and all $\boldsymbol{y} \in\left(S_{2}\right)^{|B|}$, we have

$$
z_{v}(\mathrm{P} ; A, B ; \boldsymbol{x}, \boldsymbol{y}) \leq z_{v}\left(\mathrm{P}_{p}^{\text {site }} ; A, B ; \boldsymbol{x}, \boldsymbol{y}\right),
$$

where $p=\mathrm{E}(W \bar{W})$.

For $\kappa(x, y)=x y$, this means that we have to prove that, for $|A| \geq 1$ and $|B| \geq 1$, it holds that

$$
\begin{aligned}
& \mathrm{E}\left(1-\left[1-\prod_{i=1}^{|A|}\left(1-W x_{i}\right)\right]\left[1-\prod_{j=1}^{|B|}\left(1-y_{j} \bar{W}\right)\right]\right) \\
& \quad \leq 1-\mathrm{E}(W \bar{W})\left[1-\prod_{i=1}^{|A|}\left(1-x_{i}\right)\right]\left[1-\prod_{j=1}^{|B|}\left(1-y_{j}\right)\right], \\
& \mathrm{E}\left(\prod_{i=1}^{|A|}\left(1-W x_{i}\right)\right) \leq 1-\mathrm{E}(W \bar{W})\left[1-\prod_{i=1}^{|A|}\left(1-x_{i}\right)\right] \\
& \mathrm{E}\left(\prod_{j=1}^{|B|}\left(1-y_{j} \bar{W}\right)\right) \leq 1-\mathrm{E}(W \bar{W})\left[1-\prod_{j=1}^{|B|}\left(1-y_{j}\right)\right],
\end{aligned}
$$

where respectively the cases $|A||B|>0, B=\varnothing$, and $A=\varnothing$ are considered.

Observe that, for $u \in[0,1]$ and $\boldsymbol{x} \in[0,1]^{|A|}, f(u)=1-\prod_{i=1}^{|A|}\left(1-u x_{i}\right)$ is concave and increasing by Lemma 3.2, so

$$
1-\prod_{i=1}^{|A|}\left(1-u x_{i}\right) \geq u f(1)+(1-u) f(0)=u\left(1-\prod_{i=1}^{|A|}\left(1-x_{i}\right)\right) .
$$

Similarly, for $\bar{u} \in[0,1]$ and $\boldsymbol{y} \in[0,1]^{|B|}$, we have

$$
1-\prod_{j=1}^{|B|}\left(1-\bar{u} y_{j}\right) \geq \bar{u}\left[1-\prod_{j=1}^{|B|}\left(1-y_{j}\right)\right]
$$

Replacing $u$ by $W$ and $\bar{u}$ by $\bar{W}$ in the above two equations completes the proof of Theorem 1.2.

\section{Acknowledgements}

We want to thank an anonymous referee for useful comments which led to a more elegant proof of Theorem 1.1. The presented research is supported in part by a Vici grant of the NWO (Dutch Organization for Scientific Research).

\section{References}

[1] Andersson, H. (1999). Epidemic models and social networks. Math. Scientist. 24, 128-147.

[2] Andersson, H. and Britton, T. (2000). Stochastic Epidemic Models and Their Statistical Analysis. (Lecture Notes Statist. 151), Springer, New York.

[3] Balister, P., Bollobás, B. And Walters, M. (2005). Continuum percolation with steps in the square or the disk. Random Structures Algorithms 26, 392-403. 
[4] Becker, N. G. AND StARCZAK, D. N. (1998). The effect of random vaccine response on the vaccination coverage required to prevent epidemics. Math. Biosci. 154, 117-135.

[5] Becker, N. G. And Utev, S. (2002). Protective vaccine efficacy when vaccine response is random. Biom. J. 44, 29-42.

[6] Bollobás, B., Janson, S. and Riordan, O. (2007). The phase transition in inhomogeneous random graphs. Random Structures Algorithms 31, 3-122.

[7] Britton, T., Deijfen, M. and Martin-LöF, A. (2006). Generating simple random graphs with prescribed degree distribution. J. Statist. Phys. 124, 1377-1397.

[8] Chayes, L. and Schonmann, R. H. (2000). Mixed percolation as a bridge between site and bond percolation. Ann. Appl. Prob. 10, 1182-1196.

[9] Chung, F. And Lu, L. (2002), Connected components in random graphs with given expected degree sequences. Ann. Combinatorics 6, 125-145.

[10] Cox, J. T. And Durrett, R. (1988). Limit theorems for the spread of epidemics and forest fires. Stoch. Process. Appl. 30, 171-191.

[11] Diekmann, O. and Heesterbeek, J. A. P. (2000). Mathematical Epidemiology of Infectious Diseases. John Wiley, Chichester.

[12] Grimmett, G. (1999). Percolation, 2nd edn. Springer, Berlin.

[13] Jagers, P. (1975). Branching Processes with Biological Applications. John Wiley, London.

[14] Kenah, E. and Robins, J. M. (2007). Second look at the spread of epidemics on networks. Phys. Rev. E 76, 036113, 12pp.

[15] KuUlasmaA, K. (1982). The spatial general epidemic and locally dependent random graphs. J. Appl. Prob. 19, 745-758.

[16] Miller, J. C. (2008). Bounding the size and probability of epidemics on networks. J. Appl. Prob. 45, 498-512.

[17] Newman, M. E. J. (2002). Spread of epidemic disease on networks. Phys. Rev. E 66, 016128, 11pp.

[18] Norros, I. and Reittu, H. (2006). On a conditionally Poissonian graph process. Adv. Appl. Prob. 38, 59-75.

[19] Trapman, P. (2007). On analytical approaches to epidemics on networks. Theoret. Pop. Biol. 71, $160-173$.

[20] WatTs, C. H. AND MAY, R. M. (1992). The influence of concurrent partnerships on the dynamics of HIV/AIDS. Math. Biosci. 108, 89-104.

[21] Wierman, J. C. (1994). Substitution method critical probability bounds for the square lattice site percolation model. Combinatorics Prob. Comput. 4, 181-188. 